\title{
Immunopathogenesis of psoriasis
}

\author{
Rajiv Joshi \\ Department of Dermatology, Hinduja Hospital, Mumbai, India.
}

Address for correspondence: Dr. Rajiv Joshi, 14, Jay Mahal, 'A' Road, Churchgate, Mumbai - 400020 India.

\begin{abstract}
Psoriasis is an immunologically mediated disease caused by activation of T lymphocytes that elaborate a Th1 type of immune response. Targeted manipulation of the various mechanisms involved in $\mathrm{T}$ cell activation and blockade or inactivation of the various proinflammatory cytokines released by the process of $\mathrm{T}$ cell activation are methods used in treatment of psoriasis by use of 'biologicals'.
\end{abstract}

Key Words: Psoriasis, Immunology, Pathogenesis

\section{INTRODUCTION}

Psoriasis has for long been considered a hyperproliferative skin disease with a markedly increased (5-6 times normal) rate of epidermal turnover. This, along with the invariable presence of neutrophils within the epidermis of psoriatic lesions, led to the thinking that abnormalities of the epidermis are central to the pathogenesis of psoriasis.

In the last few decades, however, this view has changed and it is now well accepted that psoriasis is in fact an immunologically mediated disease where activation of $\mathrm{T}$ lymphocytes is central to the inflammation in the dermal microenvironment and the epidermal hyperproliferation is secondary to the inflammatory events that follow a Th1 type of immune response. ${ }^{1}$ Psoriasis is in fact the most common Th1 type of immunological skin disease.

The serendipitous observation that the potent immunosuppressive drug cyclosporin A led to clearance of psoriasis spurred further research into the $T$ cell mediated pathogenesis of psoriasis and it is now known that most effective treatments of psoriasis work primarily through inhibition of $\mathrm{T}$ cell activation/ proliferation or by blocking cytokine products. The newer group of drugs in psoriasis, termed as biologicals, therefore focus on the targeted blockade of the inflammatory response induced by activated $T$ cells.

\section{T CELL FUNCTION}

In psoriasis, it is the activity of T cells that is the driving force for induction and maintenance of the skin lesions. T lymphocytes consist of functionally distinct populations of helper $T$ cells and cytolytic T cells. The principal function of $\mathrm{T}$ cells is to regulate all immune responses to protein antigens and to serve as effector cells for the elimination of intracellular microbes, e.g. mycobacteria.

T cells, unlike B lymphocytes, do not produce

How to cite this article: Joshi R. Immunopathogenesis of psoriasis. Indian J Dermatol Venereol Leprol 2004;70:10-2.

Received: December, 2003. Accepted: January, 2004. Source of Support: Nil. 
antibodies but recognize only processed peptide antigens that are attached to proteins encoded by the MHC class II genes. Therefore, for activation, T cells need antigen presenting cells (APCs) to process and present peptide fragments on the APC cell surface. T cells respond to only cell surface associated antigens and not to soluble antigens. On appropriate stimulation, $\mathrm{T}$ cells secrete various lymphokines. T cells may also inhibit immune responses and are then known as suppressor T cells.

Functionally different populations of $T$ cells express distinct cell membrane proteins that serve as phenotypic markers for different lymphocyte populations. Most helper T cells express CD 4, while cytolytic and suppressor cells are CD 8 positive. CD (cluster of differentiation) refers to a molecule on the cell surface that is recognized by a cluster of monoclonal antibodies and which can be used to identify the lineage of or the stage of differentiation of lymphocytes.

\section{T CELL ACTIVATION}

Activation of $\mathrm{T}$ cells requires three steps ${ }^{1}$ : binding; antigen specific activation, also known as signal 1; and non-antigen specific cell-cell interaction, known as signal 2 .

\section{Binding}

The $T$ cell attaches to the antigen presenting cell through surface adhesion molecules which are located reciprocally on the cell surfaces of both the $T$ cells and the APCs. LFA-1 (leucocyte function associated antigen 1) and CD2 are adhesion molecules of T lymphocytes that attach to reciprocating adhesion molecules expressed on the surface of antigen presenting cells (intercellular adhesion molecule, ICAM-1; and LFA-3). In skin the Langerhans cell is the most efficient antigen presenting cell.

\section{Antigen specific activation}

Once the T cell-APC binding has occurred through their respective surface adhesion molecules, the antigen is presented to the T cell by the APC. T cells express the $T$ cell receptor (TCR) which recognizes the peptide antigen being presented by the APC in the groove of the MHC complex. This antigen stimulated activation leads to conversion of the naïve $T$ cell into an antigen specific cell that may further develop into a long lived memory cell that circulates in the body and can recognize the same antigen at a later date, even after several years.

\section{Non-antigen specific cell-cell interaction}

This is also known as co-stimulation. If co-stimulation by other cell surface molecules does not occur following antigen presentation, the $T$ cell will not respond to the antigen and will undergo apoptosis or be rendered unresponsive to that antigen in the future (anergy).

\section{EFFECTOR FUNCTIONS OF ACTIVATED T CELLS} (Secretion of cytokines and magnification of the immunologic cascade)

Once the T cell is activated the next step is induction of inflammatory responses and tissue changes leading to the clinical picture of psoriasis. Many cell types are involved in this final step ( $\mathrm{T}$ cells, macrophages, dendritic cells, vascular endothelium and keratinocytes). These secrete various cytokines that promote and maintain tissue inflammation. In psoriasis it is likely that a cascade of cytokines are secreted by different cells in the local microenvironment and play a role leading to the typical phenotypic responses seen in psoriasis, namely, vascular dilatation, dermal inflammation and resultant hyperproliferation of the epidermis. ${ }^{1}$ The cytokines involved in the development of psoriasis include granulocyte-macrophage colony stimulating factor (GMCSF), epithelial growth factor (EGF), IL8, IL12, IL1, IL6, interferon-gamma, tumor necrosis factor-alpha (TNF- $\alpha$ ). The effects of these cytokines include keratinocyte proliferation, neutrophil migration, potentiation of Th1 type of responses, angiogenesis, upregulation of adhesion molecules and epidermal hyperplasia.

TNF- $\alpha$ is strongly implicated in the pathogenesis of psoriasis and psoriatic arthritis. It plays a critical role in activation of innate and acquired immune responses leading to chronic inflammation, tissue damage and keratinocyte proliferation. TNF- $\alpha$ functions in a positive feedback loop by recruiting more inflammatory cells and upgrading receptors on those cells. TNF- $\alpha$ levels 
are markedly increased in skin lesions, synovium and serum of patients with psoriasis and these correlate with the severity of the disease. Decreased levels are associated with clinical resolution.

\section{BIOLOGICALS IN PSORIASIS ${ }^{2-5}$}

With this background of immunologic dysfunction in psoriasis, various methods for targeted 'biologic' treatment of psoriasis have emerged. These include four basic strategies:

1. Decrease the number of effector (activated) T cells.

2. Inhibit cell-cell interaction that leads to $T$ cell activation and migration of $\mathrm{T}$ cells to the skin.

3. Change the cytokine profile from Th1 $\rightarrow$ Th2 (immune deviation).

4. Bind and inactivate proinflammatory cytokines to prevent effects on keratinocytes and downregulate the inflammatory process.

Of all these, inhibition of cell-to-cell interaction to prevent $\mathrm{T}$ cell activation and blockade or inactivation of cytokines, especially TNF- $\alpha$, are the approaches taken by the various biologicals that are currently available for the treatment of moderate to severe psoriasis (Table 1).

\section{CAUSES OF T CELL ACTIVATION}

While much is known about the immune responses in psoriasis once $\mathrm{T}$ cell activation occurs, there remain lacunae in our understanding of the primary causes that lead to $T$ cell activation. Why $T$ cells get activated in psoriasis in the first place and which are the antigens that cause their activation is not known. While streptococcal antigens that work by molecular mimicry (in guttate psoriasis) and superantigens of various bacterial species are being considered, this is an area that requires further research. The answers may give our patients of psoriasis hope that their disease may be prevented rather than just controlled.

In sum, psoriasis is an immunologically mediated disease caused by activation of $\mathrm{T}$ lymphocytes that elaborate a Th1 type of immune response. Targeted
Table 1: Mechanisms of action of Immunomodulators in psoriasis

\section{Current therapies:}

1. Methotrexate: Inhibits replication of $T$ and $B$ lymphocytes and suppresses secretion of various cytokines like IL1 (interleukin 1), interferon-gamma and TNF-alpha.

2. Cyclosporin $\mathbf{A}$ : Inhibits $T$ cell activation by inhibiting interleukin 2 (IL 2) and interferon gamma production through inhibition of calcineurin.

3. Mycophenolate mofetil: Inhibits lymphocyte proliferation and downgrades adhesion molecules that are activated in response to antigen stimulation.

4. Phototherapy: Causes alteration of antigen presenting cell population (Langherhans cells), modifies intra and intercellular signaling mechanisms leading to development of Th2 responses in preference to Th1 responses. It also causes apoptosis of activated T lymphocytes.

\section{Future biologicals:}

1. Alefacept: Inhibits $T$ cell activation and proliferation by binding to CD-2 on T cells and blocking the CD-2:LFA-3 interaction. (blocks costimulatory signal 2).

2. Efalizumab: It is anti-CD-11a which is the alpha subunit of LFA1. Efalizumab blocks LFA-1:ICAM-1 interaction and prevents activation of $T$ cells (blocks the first step of binding of $T$ cells and antigen presenting cells).

3. Etanercept: Competitive inhibitor of tumor necrosis factor-alpha. It binds to TNF-alpha and inactivates it, preventing its proinflammatory effects.

4. Infliximab: Mouse antibody to TNF-alpha. Similar effects as etanercept in blocking effects of TNF-alpha.

manipulation of the various mechanisms involved in $\mathrm{T}$ cell activation and blockade or inactivation of the various proinflammatory cytokines released by the process of $T$ cell activation are methods used in the treatment of psoriasis by the use of 'biologicals'.

\section{REFERENCES}

1. Mehlis SL, Gordon KB. The immunulogy of psoriasis and biological immunotherapy. J Am Acad Dermatol 2003;49: (Suppl):S44-50

2. Miller GT, Hochman PS, Meier W, Tizard R, Bixlor SA, Rosa $M D$, et al. Specific interaction of lymphocyte function associated antigen 3 with CD2 can inhibit T cell responses. J Exp Med 1993;178:211-22.

3. Krueger J, Gottlieb A, Miller B, Dedrick R, Garovoy M, Walicke P. Anti CD 11 treatment for psoriasis increases circulating $T$ cells and decreased plaque $T$ cells, consistent with inhibition of cutaneous T cell trafficking. J Invest Dermatol 2000;115:333.

4. Peppel K, Crawford D, Beutler B. A tumour necrosis factor receptor $\operatorname{IgG}$ heavy chain chimeric protein as a covalent antagonist of TNF activity. J Exp Med 1991;174:1483-9.

5. Chaudhari U, Romano P, Mulcahy LD, Dooley LT, Baker DG, Gottlieb AB. Efficacy \& safety of Infliximab monotherapy for plaque type psoriasis. Lancet 2001;357:1842-7. 